\title{
Fungating carcinoma of the stomach: en bloc multiple organ resection and abdominal wall reconstruction
}

\author{
Kent-Man Chu, David TM Lai, Harvey S Stern, Donald M Sheldon
}

\begin{abstract}
Summary
A patient with carcinoma of the stomach invading multiple adjacent organs and fungating through the anterior abdominal wall was treated by en bloc multiple organ resection and abdominal wall reconstruction. The patient is alive and well at the time of writing, six months after the operation. The rationale for embarking on multiple organ resection for gastric cancer is discussed. However, such an aggressive surgical approach should only be applied to carefully selected patients who are medically fit and have no evidence of widespread systemic metastases.
\end{abstract}

Keywords: gastric cancer, multiple organ resection

As many as $\mathbf{5 0} \%$ of all gastric cancers infiltrate neighbouring organs. ${ }^{1}$ It is obvious, therefore, that surgical extirpation of the disease entails more than just gastrectomy. McNeer and associates were probably amongst the first to advocate gastrectomy and en bloc resection of invaded organs with the hope of extending survival. ${ }^{2}$ This attitude was supported by subsequent reports on long-term survivors after en bloc resection of invaded organs. ${ }^{3,4} \mathrm{We}$ report a patient with a rare presentation of carcinoma of stomach treated by en bloc multiple organ resection and abdominal wall reconstruction.

\section{Case report}

Royal Prince Alfred

Australia

Department of Upper

Gastrointestinal

Surgery

K-M Chu

DTM Lai

DM Sheldon

Department of Plastic

and Reconstructive

Surgery

HS Stern

Correspondence to Mr Donald M Sheldon, Royal Prince Alfred Hospital Medical Centre, 100 Carillon Avenue, NSW 2050,

Australia

Accepted 30 November 1994

A 61-year-old woman was admitted to the Department of Upper Gastrointestinal Surgery and Total Parental Nutrition at the Royal Prince Alfred Hospital, Sydney, with a fungating mass in the left upper abdomen. She had been enjoying good health until six months earlier when she gave a history of recurrent formation of painful small abscesses in the left upper abdomen. Initially, these abscesses healed individually after discharging some clear serous fluid. Gradually, they became uncontrolled and coalesced to form larger abscesses. There was no history of overt gastrointestinal bleeding or malaena. She had no abdominal pain or vomiting. She had a weight loss of about $5 \mathrm{~kg}$ in the past three months to the present admission, she was found to be profoundly anaemic with a haemoglobin level of $4.8 \mathrm{~g} / \mathrm{dl}$ necessitating transfusion at a district hospital. No further investigation was instituted. A rapidly growing fungating mass had been evident in the left upper abdomen for the previous month. Her past medical history was unremarkable. She had no past history of abdominal surgery.

On examination, she looked a bit wasted. She had pallor but no jaundice. There was no palpable lymph node. She had no ankle oedema. She was afebrile with a normal blood pressure. Her abdomen was not distended although there was an obvious fungating lesion in the left upper quadrant. The lesion measured about $10 \mathrm{~cm}$ in diameter. The overlying skin was erythematous with areas of ulceration. Deep to the skin lesion was a palpable abdominal mass. The mass was hard in consistency. There was no hepatomegaly. Rectal examination and chest X-ray were normal. Serum electrolytes, urea, creatinine and liver enzymes were all within normal limits. Serum albumin was low at $32 \mathrm{~g} / \mathrm{l}$. Haemoglobin was $7.3 \mathrm{~g} / \mathrm{dl}$, white cell count $18.3 \times 10^{9} / 1$ and platelet count $510 \times 10^{9} / 1$. She was transfused. Gastroscopy had revealed diffuse tumour involvement of virtually the whole body of stomach. Computed tomography (CT) of the abdomen (figure) revealed a massive tumour of

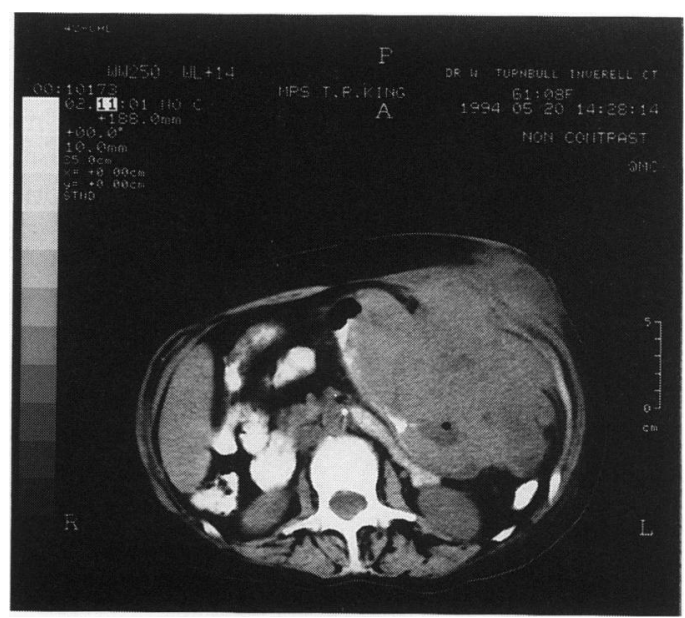
because of early satiety. About six weeks prior
Figure Abdominal CT scan of the patient showing bulky tumour fungating through the abdominal wall. There was no evidence of ascites, para-aortic lymphadenopathy or liver metastases 
the stomach invading the anterior abdominal wall. There was no ascites, no para-aortic lymphadenopathy and no liver metastases noted.

Laparotomy revealed that the tumour in the stomach was invading the anterior abdominal wall, segment III of the liver, the distal pancreas, splenic hilum and transverse colon. There was no liver metastasis, no metastatic peritoneal disease and no distal lymph node metastasis noted. Total gastrectomy with en bloc distal pancreatectomy, splenectomy, partial wedge resection of segment III of liver, transverse colectomy and left upper abdominal wall resection was performed. Intestinal continuity was attained by Roux-en-Y oesophagojejunostomy. The abdominal wall defect $(18 \times 12 \mathrm{~cm})$ was temporarily covered by omentum. The defect was subsequently reconstructed with Marlex mesh covered by latissimus dorsi free flap and split thickness skin grafting one week afterwards. She made an uneventful recovery. Total parenteral nutrition was given for nutritional support. She could take normal oral feeding three weeks after the first operation. The abdominal wall reconstruction healed well. She is alive and well six months after the operation.

Histology of the resected specimen revealed a moderately differentiated intestinal-type adenocarcinoma arising from the stomach. The tumour showed extensive necrosis and invaded the transverse colon, the peripancreatic fat, the anterior abdominal wall and skin, with focal epidermal ulceration. No lymph nodes could be definitely identified as most of the fatty tissue included with the specimen had been infiltrated by the tumour.

\section{Discussion}

In a study of patients who had survived for more than 10 years after resection for carcinoma of the stomach, ${ }^{3}$ Remine and colleagues reported five long-term survivors in whom the tumours had invaded the colon, liver or pancreas. It became apparent that significant survival benefit could be attained by resecting locally advanced tumours of the stomach.

In order to define which patients would benefit from multiple organ resection, a retrospective study ${ }^{4}$ was done on 72 patients who had had a gastrectomy and en bloc resection of invaded organs. For comparison, 266 patients who had undergone gastrectomy alone for carcinoma of the stomach during the same period were studied as a control group. The 5-year survival of the patients with en bloc resection was only $7 \%$ which was significantly lower than $18 \%$ of the control group $(p<0.01)$. However, when a separate analysis was made of the patients who had early stage carcinoma (T4 N0 and T4 N1 compared with T1-3 NO and T1-3 N1 of the control), the 5 -year survival of the study and control groups were not statistically significantly different. Therefore, in the absence of detectable systemic metastases, aggressive resection of these tumours seems to have been warranted in carefully selected patients. A large tumour

\section{Learning points}

- as many as $50 \%$ of all gastric cancers infiltrate neighbouring organs

- complete en bloc resection of invaded organs has a beneficial effect on survival, provided that there is no evidence of peritoneal dissemination, liver metastasis, or widespread nodal involvement

- such an approach is feasible in a patient with a fungating tumour although careful patient selection is important

which might have invaded one or more adjacent organs or structures should not be assumed incurable. ${ }^{4}$

A more recent study ${ }^{5}$ on 281 patients with gastric carcinoma invading adjacent organs confirmed the beneficial effect on survival of en bloc complete excision of invaded organs, irrespective of the number or site of organs involved, provided that there was no evidence of 'incurable factors' (peritoneal dissemination, liver metastasis and widespread nodal involvement).

The patient in the present report had a locally advanced tumour infiltrating adjacent organs and fungating through the skin. Without treatment, the patient was going to suffer from the pain, infection and discharges from the fungating tumour. Her feeding was going to be a problem with the tumour nearly replacing the stomach. As she had no clinical evidence of systemic metastasis or widespread nodal involvement, the option of total gastrectomy with mutiple organ and abdominal wall resection would provide a fair chance of disease control even though the primary aim was palliation. The availability of abdominal wall reconstruction certainly helped in dealing with the large abdominal wall defect as a result of such a surgical approach. Both tensor fasciae latae flap and latissimus dorsi flap have been advocated for reconstructing abdominal wall defects after resection for malignancy involving the abdominal wall. ${ }^{6,7}$

The present report illustrates that surgery may be beneficial in locally advanced carcinoma of the stomach. Such an aggressive surgical approach, however, should not be undertaken as a routine. It should only be performed on carefully selected patients who can withstand the procedure and who have no evidence of widespread systemic metastases.

Involvement of skin in carcinoma of stomach is rare. An early report ${ }^{8}$ gave an incidence of roughly $2 \%$, although the nature of the skin involvement (direct infiltration or metastatic) was not defined. Carcinoma of the stomach with metastases to the skin in the form of cancer-en-cuirasse and metastatic skin nodules had also been reported in the literature. ${ }^{9,10}$ In the reports demonstrating the survival benefit of en bloc resection of invaded organs or structures, the abdominal wall was not included the list of invaded structures. Whether resection of invaded abdominal wall confers the same survival benefit remains to be seen in the present patient. It is, however, interesting to 
note that prolonged survival has been reported after extended resection in a case of colonic

1 Wanke M, Schwan $H$. Pathology of gastric cancer. World $\mathcal{f}$ Surg 1979; 3: 675-84.

2 McNeer G, Sunderland DA, McInnes G, et al. A more thorough operation for gastric cancer; anatomic basis and description of technique. Cancer 1951; 4: 957.

3 Remine WH, Gomes MMR, Dockerty MB. Long term survival (10 to 56 years) after surgery for carcinoma of survival (10 to 56 years) after surgery
stomach. Am $₹$ Surg 1969; 117: $177-84$.

4 Papachristou DN, Shiu MH. Management by en bloc multiple organ resection of carcinoma of the stomach multiple organ resection of carcinoma of the stomach invading

483-7.

5 Korenaga D, Okamura T, Baba H, Saito A, Sugimachi K. Results of resection of gastric cancer extending to adjacen organs. Br $\mathcal{f}$ Surg 1988; 75: $12-5$.

6 O'Hare PM, Leonard AG. Reconstruction of major abdominal wall defects using the tensor fasciae latae myocutaneous flap. Br $\mathcal{F}$ Plast Surg 1982; 35: 361-6. cancer requiring abdominal wall reconstruction. ${ }^{11}$

7 Neven P, Shepherd JH, Tham KF, Fisher C, Breach N. Reconstruction of the abdominal wall with a latissimus dorsi musculocutaneous flap: a case of a massive abdominal wall metastasis from a cervical cancer requiring palliative resection. Gynecol Oncol 1993; 49: 403-6.

8 Olearchyk AS. Gastric carcinoma. A critical review of 243 cases. Am $¥$ Gastroenterol 1978; 70: 25-45.

(abs. Am g Gastrontero Abramovich IaA, Bul 'vakhter LA, Kripal 'skii LN. [A case of metastatic cancer en cuirasse of the skin in stom

10 Choi HM, Myung KB, Kook HI. Cutaneous metastatic adenocarcinoma of stomach-nodular and inflammatory carcinoma. $\mathcal{F}$ Korean Med Sci 1986; 1: 49-52.

11 Gardner B. Five-year survival after extended resection of colon cancer. $\mathcal{f}$ Surg Oncol 1987; 34: 258-61.

\title{
Fatal bronchospasm after oral ingestion of isphagula
}

\author{
DC Hulbert, PJ Thorpe, AJ Winning, MW Beckett
}

\section{Summary \\ We report the case of a 40-year-old asthmatic who suffered fatal broncho- spasm following oral injestion of a lax- ative containing isphagula.}

Keywords: asthma, bronchospasm, isphagula

We describe a case of fatal bronchospasm following oral ingestion of isphagula. It is well established that bronchospasm associated with occupational asthma can occur after inhalation of isphagula and that anaphylaxis can occur after oral ingestion of isphagula and psyllium. However, fatal anaphylaxis after oral ingestion of isphagula has never been reported.

\section{Case report}

West Middlesex

University Hospital,

Twickenham Road, Isleworth, Middlesex

TW7 6AF, UK

Department of

Accident and

Emergency

DC Hulbert

MW Beckett

Department of

Pathology

PJ Thorpe

Department of Chest

Medicine

AJ Winning

Accepted 16 November 1994
A 40-year-old man, known to be asthmatic, was taken to the Accident and Emergency department as a priority call. He was first diagnosed as being asthmatic in 1986 and his symptoms were controlled with inhaled salbutamol and beclamethasone diproprionate.

He had had two previous hospital admissions, the first precipitated by the ingestion of a single tablet of aspirin, the second by ibuprofen. On both occasions he had severe bronchospasm requiring admission to the Intensive Care Unit, although ventilation was not required.

According to his wife, the evening prior to admission he had felt wheezy and had taken nebulised salbutamol at home.
In the morning he had no respiratory symptoms but had taken Fybogel as he had been unable to open his bowels for several days. $\mathrm{He}$ had previously taken this with no abnormal sequaelae. When his wife left for work at $07.15 \mathrm{~h}$ he was entirely well. Following the ingestion of one sachet $(3.5 \mathrm{~g})$ between $07.30 \mathrm{~h}$ and $09.30 \mathrm{~h}$ he became acutely short of breath and was noted by his family to be wheezy. $\mathrm{He}$ took nebulised salbutamol which had no effect and an ambulance was called. He then had a cardiorespiratory arrest and, in spite of full resuscitative measures in the Accident and Emergency Department, he died at $11.00 \mathrm{~h}$.

At post-mortem examination the epiglottis and larynx were normal; the upper airways contained a small amount of mucus and the lungs were found to be severely congested with blood with evidence of acute pulmonary oedema. It was thought that these findings were consistent with acute hypoxia due to sudden onset of severe bronchospasm.

\section{Discussion}

Fybogel is a laxative preparation, the main constituent being isphagula husk. This itself is derived from the dried seeds of Plantago ovata, and there is evidence that this can induce asthma, bronchospasm, and even anaphylaxis. ${ }^{1}$

Psyllium (USP) which is used as a laxative in America is derived from a combination of the dried seeds of Plantago ovata and Plantago psyllium and this has also been shown to cause bronchopasm.

Sensitisation probably occurs through 